\title{
Meningiomas de la base de cráneo. Un sistema predictivo para conocer las posibilidades de su extirpación y pronóstico
}

\author{
F. Morales*; A. Maillo*; A. Díaz-Álvarez**; M. Merino*; A. Muñoz-Herrera***; J. Hernández* y D. Santamarta*
}

Servicio de Neurocirugia. Hospital Universitario de Salamanaca.

Resumen

Objetivo. Encontrar un sistema predictivo que basándose únicamente en datos preoperatorios nos oriente, con fiabilidad, sobre : $1^{\circ}$ qué meningiomas de la base de cráneo pueden ser extirpados total o parcialmente y $2^{\circ}$ cual es su pronóstico postquirúrgico.

Métodos. Se ha revisado de forma retrospectiva, las historias clínicas e iconografías de 85 pacientes, con meningiomas de la base de cráneo, intervenidos entre 1990 al 2002. De ellas se han tomado nueve variables que han sido tratadas estadísticamente, mediante un test estándar, para correlacionarlas con: $1^{\circ}$ resección total o parcial del tumor (Simpson, 5 grados) y $2^{\circ}$ conocido el mencionado grado de resección total o parcial, éste se correlacionó con el pronóstico funcional postoperatorio del paciente (Karnofsky).

Resultados. De las variables preoperatorios analizadas, solo dos demostraron tener una asociación significativa con la extirpación total o parcial del tumor, así como con el pronóstico postquirúrgico del paciente. Dichas variables fueron: a) las arterias englobadas por el tumor $(p=0.001)$ y b) la afectación de pares craneales $(p<0.0001)$. Seguidamente, se conjugaron las diversas posibilidades de cada una de ellas para conocer el grado de extirpación tumoral (total o parcial). Para predecir el pronóstico funcional postoperatorio del enfermo, se relacionó la extirpación total o parcial con la escala de Karnofsky. De ambos análisis, grado de extirpación y pronóstico, se obtuvo el siguiente sistema de gradación:

Grado I: meningiomas, de la base de cráneo, que no afectan a pares craneales ni engloban arterias o bien solamente afectan a un par craneal o engloban sólo una arteria.

En ellos las posibilidades de extirpación total se encuentran en un $98,3 \%(p<0.0001)$ y las perspectivas de alcanzar 70 puntos en la escala de Karnofsky son de un $96,5 \%$.

Grado II: meningiomas que afectan a un par cra-

Recibido: 14-10-04. Aceptado: 17-03-05 neal e incluyen dos o más arterias. La extirpación total en estos casos disminuye al $83,3 \%(p<0.0001)$ y las perspectivas de alcanzar 70 puntos en la escala de Karnofsky descienden a un $70,6 \%$.

Grado III: meningiomas que afectan dos o más pares craneales e incluyen varias arterias. En este grupo, las posibilidades de una extirpación total son de un $42,9 \%(p<0.0001)$ con las perspectivas de alcanzar los 70 puntos en la escala de Karnofsky en sólo el $60 \%$.

Conclusiones. El sistema de gradación que se propone permite, en los casos de meningiomas de la base de cráneo: $1^{\circ}$ predecir las posibilidades de una extirpación total o parcial y $2^{\circ}$ conocer el pronóstico quirúrgico de los pacientes en cada grupo. Aplicando este sistema podremos reducir nuestra morbilidad quirúrgica, anticipar la necesidad de una terapia con radiocirugía y dar una información, más precisa, al paciente y familiares sobre nuestras propuestas quirúrgicas.

PALABRAS CLAVE : Meningiomas. Base de cráneo. Gradación extirpación quirúrgica.

Skull base meningiomas: a predictive system to know the extent of their surgical resection and patient outcome

Summary

Objective. The aim of this study was to build a preoperative predictive system which could provide reliable information about: $1^{\circ}$ which skull base meningiomas can be total or partially removed, and $2^{\circ}$ their surgical outcome.

Method. Patient histories and imaging data were reviewed retrospectively from 85 consecutive skull base meningiomas patients who underwent surgery from 1990 and 2002. From the preoperative data, nine variables were selected for conventional statistical analysis as regards their relationship with: $1^{\circ}$ total vs partial tumor resection and $2^{\circ}$ with patients outcome according to the degree of tumour removal. 
Results. From the nine variables analysed only two had a statistical association with the type of tumour resection performed (total vs partial) and the patient outcome: 1) arteries encasement and 2) cranial nerves involvement. Upon correlating these two variables with the type of tumour resection performed (total vs partial) and with the Karnofsky'scale to evaluate patients surgical outcome, the following grading groups were identified:

Grade I: skull base meningiomas which did not involve cranial nerves or artery or only encased one artery or one cranial nerve. In these cases the incidence of gross tumour resection was $98,3 \%(p<0.0001)$ and the perspective to reach 70 points in the Karnofsky'scale was of $96,5 \%(p=0.001)$.

Grade II: skull base meningiomas which involved one cranial nerve and encased, at least, two main cerebral arteries. In these cases, the frequency of total resection, decreased to $83,3 \%(p<0.0001)$ and the probability to reach 70 points in the Karnofsky'scale was $70,6 \%$ $(p=0.001)$.

Grade III: skull base meningiomas which involved two or more cranial nerves and encased several arteries In this group, the frequency of a total resection was of $42,9 \%(p<0.0001)$ and the probability of reaching 70 points in the Karnofsky'scale was only $60 \%(p=0.001)$.

Conclusions. We propose a preoperative grading system for skull base meningiomas that helps predicting both whether total or partial tumor removal will be achieved during surgery and the immediate postsurgical outcome of the patient. In applying this predictive system we will be able to reduce surgical morbidity, to advance the possibility of a radiosurgical treatment and give a more precise information to the patients and their families about our surgical decision-making process.

KEY WORDS : Skull base. Meningiomas. Grading predicting system, Outcome.

\section{Introducción}

Los meningiomas que asientan en la base del cráneo, son por lo general tumores que, en su tratamiento, presentan serias dificultades a la hora de planificar una estrategia quirúrgica. Para algunos autores, la localización y/o el tamaño de estos procesos ${ }^{6,20}$ desempeñan un importante papel en la toma de la decisión quirúrgica. Para otros, la afectación de pares craneales o bien la incorporación intratumoral de una arteria principal o incluso la existencia de una invasión ósea son considerados como factores que juegan una mayor influencia a la hora de la planificación quirúrgica ${ }^{3,4,5,16,17,18,19}$. Cualquiera que sea el factor considerado, es de interés recordar que la mayoría de estos tumores son benignos, pero con propensión a las recidivas ${ }^{1,69,13,21}$, por lo que una extirpación lo más radical posible debería ser la meta principal ${ }^{1,2,4,7,21,23,27}$.

Actualmente, el criterio que predomina en el tratamiento de estos meningiomas es la experiencia del cirujano. Dependiendo de ello, lo que para unos podría ser una cirugía radical ${ }^{2,47}$ para otros podría ser una indicación de tratamiento mediante radiocirugía ${ }^{8,10,11,15,20,22}$. Esta situación ha creado un estado de confusión que hace difícil conocer cual es la realidad o el mito sobre el tratamiento de estos procesos expansivos.

Sea uno u otro el criterio, la solución podría estar en tener unos puntos de referencia que, al igual que sucede con las escalas existentes para el pronóstico de los traumatismos craneales $^{26}$, hemorragias subaracnoideas ${ }^{12}$ o malformaciones arteriovenosas ${ }^{25}$, nos pudieran servir de base para la toma de nuestras decisiones quirúrgicas. Con esta idea nos hemos propuesto alcanzar los siguientes objetivos: 1. Conocer si por los estudios preoperatorios de exploración neurológica y neuroimagen, se puede predecir qué meningiomas de la base de cráneo podrían ser extirpados de forma total o parcial. 2. Establecer el pronóstico de estos pacientes, según el grado de resección tumoral (parcial o total) y 3 . Si con estos datos se puede construir un sistema de valoración pronóstica.

\section{Material y métodos}

Para responder a nuestros objetivos hemos revisado, todos los meningiomas intervenidos en el Servicio de neurocirugía del Hospital Universitario de Salamanca desde 1990 a 2002, seleccionándose 85 pacientes, cuyos meningiomas asentaron en la base de cráneo.

Se realizó una base de datos en la que se incluyeron nueve variables, todas tomadas de los estudios preoperatorios. Dichas variables, desde el punto de vista de la exploración neurológica, fueron: edad, sexo, clínica de los pares craneales y estado funcional preoperatorio de los pacientes, este último evaluado mediante la escala de Karfsnosky ${ }^{14}$. El resto de las variables, localización tumoral, tamaño del proceso, arterias englobadas por la tumoración, invasión ósea y multiplicidad se obtuvieron del examen de las imágenes. En nuestra serie, ningún caso ha sido tratado previamente en otro Hospital ni quirúrgica ni mediante radiocirugía u otro tipo de terapia.

El resultado de estas variables se relacionó: $1^{\circ}$ con la resección total o parcial del tumor (Simpson, V grados; en esta serie, se ha considerado extirpación total Ios grados I, II y III) y $2^{\circ}$ el grado de resección, total o parcial, con el pronóstico funcional postoperatorio del paciente (escala de Karnofsky). La evolución posquirúrgica de cada enfermo ha sido revisada como mínimo durante un año, con controles de los posibles cambios en la clínica e imagen, cada 6 
meses.

La Cirugía de estos enfermos fue llevada a cabo, principalmente, por los dos primeros autores de este trabajo y su intención fue la de realizar siempre una extirpación total de la tumoración. Hubo varios casos en los que para conseguir dicha meta tuvimos la necesidad de extirpar la tumoración en varios tiempos quirúrgicos. En un voluminoso meningioma del ángulo pontocerebeloso, el paciente fue intervenido hasta en cuatro ocasiones, en un plazo de seis meses, para realizar una progresiva, pero total, extirpación y poder respetar los pares craneales. En estos casos y desde el punto de vista estadístico, las diversas cirugías fueron consideradas como una y su resultado final como resección total o parcial. Referente a la escala de Karnofsky, se ha tomado como referencia la cifra de 70 puntos al considerarse que quien alcanza dicha puntuación representa a una persona con autonomía en su vida diaria.

El análisis estadístico, de todas las variables, incluidas en este trabajo, se realizó con el programa informático SPSS 11.0 ( Software Programs, Chicago, II.). Con el fin de establecer la significación estadística de las diferencias observadas entre grupos, se usaron los test, $t$ de Student y Mann-Whitney U, para variables continuas, dependiendo de que éstas presentaran una distribución normal o no. Para variables cualitativas se usó la prueba de chi-cuadrado de Pearson (cross-tab; SPSS).

Para predecir las variables asociadas con el tipo de extirpación, se realizó un análisis multivariante mediante la regresión logística de Cox. En todos los casos se consideró que existían diferencias estadísticas significativas cuando se observaron valores de $\mathrm{p}<0.05$

\section{Resultados}

Desde el periodo de 1990 al 2002, se intervinieron 236 pacientes con meningiomas intracraneales de los que 85 $(36 \%)$ se localizaron en la base del cráneo. La edad media, de estos 85 pacientes fue de 56,8 años (rango: 13-81 años) encontrándonos con $63(74,1 \%)$ mujeres y $22(25,9 \%)$ hombres.

La localización de estos 85 meningiomas fue la siguiente : fosa anterior $29(34,1 \%)$, fosa anterior y media $28(32,9 \%)$, fosa media y senocavernoso $4(4,7 \%)$ y fosa posterior $24(28,3 \%)$.

En la serie que presentamos se consiguió una extirpación total de la tumoración en el 90,6\% de los casos (grados I, II y III de Simpson). En el grado I $=5,9 \%$, en el grado II $=$ $70,6 \%$ y en el grado III $=14,1 \%$. Una extirpación parcial se efectuó en el 9,4\% (grado IV de Simpson). En ningún caso se concibió solamente una craneotomía descompresiva, (grado V de Simpson), como tratamiento del proceso.

De las nueve variables preoperatorias analizadas demostraron tener una asociación estadísticamente significativa, con la posibilidad de realizar una extirpación tumoral total o parcial las siguientes: la localización del meningioma $(\mathrm{p}=0.03)$, la inclusión de arterias en la tumoración $(\mathrm{p}=$ $0.001) \mathrm{y}$, sobre todo, la afectación de los pares craneales $(\mathrm{p}<0.0001)$.

\section{Grado de extirpación}

Conociéndose que las variables más significativas, para determinar el grado de extirpación tumoral, la constituyan las arterias y pares craneales atrapados por la tumoración, se conjugaron las diversas posibilidades de cada una de ellas de la siguiente forma:

1. A la no afectación de pares craneales, se le dio 0 puntos; a la afectación de un solo par, 1 punto y a la afectación de más de un par, 2 puntos.

2. Referente a la inclusión de las arterias dentro de la tumoración, si el meningioma no envolvía ninguna arteria, la puntuación fue de 0 , pero si encerraba una o más arterias, se le dio 1 punto.

Los resultados de estas combinaciones fueron las siguientes: $1^{\circ}$ si el meningioma no englobaba pares craneales ni arterias o sólo a un par craneal o una arteria, las posibilidades de extirpación total fueron de un $98,3 \%$ $(\mathrm{p}<0.0001) 2^{\circ}$ si el meningioma incluye a un solo par craneal y a una o mas arterias, las posibilidades de una extirpación total descendieron a un $83,3 \%(\mathrm{p}<0.0001)$ y $3^{\circ}$ dichas posibilidades de extirpación total cayeron de forma llamativa a un $42,9 \%(\mathrm{p}<0.0001)$ si la tumoración incluye dos o más pares craneales y arterias. En dicho estudio se puede observar que existe una clara relación lineal inversa entre la capacidad de englobar elementos vasculoneurales por la tumoración y las posibilidades de que ésta sea totalmente extirpada. A más elementos incluidos en el tumor, menos posibilidades de que éste pueda ser radicalmente extirpado.

Es, también, de interés destacar que las posibilidades de extirpación total cambian dependiendo de si en la inclusión tumoral sólo hay arterias o se observa además pares craneales. La afectación de pares craneales por la tumoración condiciona la extirpación total de la misma, más que si el meningioma englobara sólo arterias. Esto se explica porque la disección de estas últimas es más accesible debido tanto a su consistencia como a detectarse más fácilmente por su latido.

\section{Pronóstico del paciente y grado de extirpación tumoral}

Para conocer si el grado de resección tumoral tenía algún valor predictivo con respecto al pronóstico de los enfermos, se analizó, en el postoperatorio, la escala funcional de Karnofsky con el tipo de cirugía practicada: extirpación total o parcial. De dichas combinaciones, se 


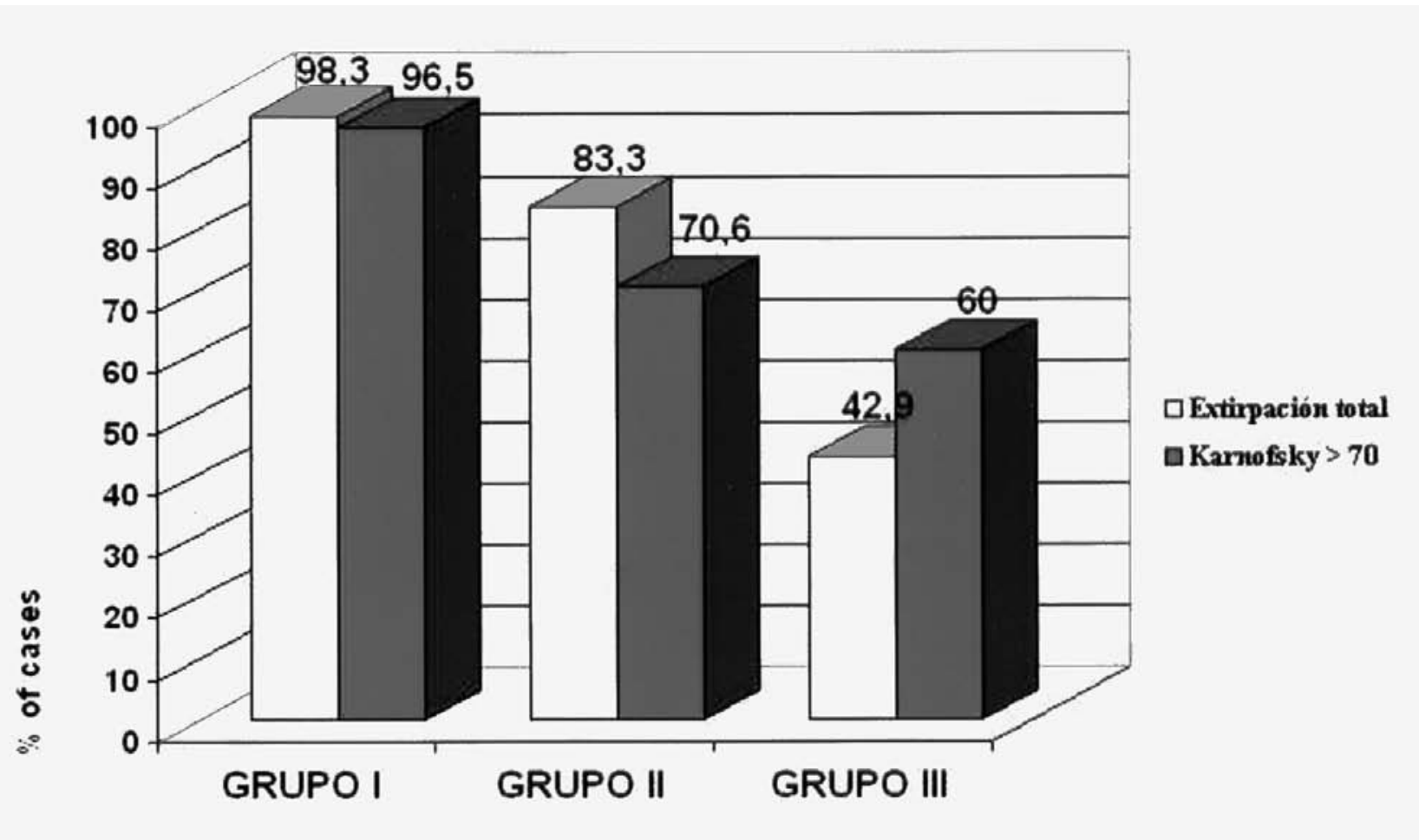

Figura 1. Resultados obtenidos en porcentajes de extirpación total (Simpson I, II, III) y calidad funcional postoperatoria (Karnofsky > 70 puntos) en 85 meningiomas localizados en la base de cráneo de acuerdo con los diferentes grupos realizados en función de la afectación de pares craneales y/o estructuras arteriales.

obtuvieron lo siguientes resultados:

1. En los paciente que se realizó una resección total, el $96,5 \%$ alcanzó 70 puntos o más en la escala de Karnofsky y sólo el 8,3\% tuvo una puntuación inferior a $70(\mathrm{p}=0.01)$.

2. En los pacientes cuya extirpación tumoral fue parcial, sólo el 42,9\% de casos obtuvo una puntuación de 70 ( $\mathrm{p}=$ $0.01)$.

Según muestran estos resultados a mayor resección tumoral, mejor pronóstico del paciente, si bien hay que hacer la importante aclaración de que dicha resección está en función de los resultados del apartado anterior, es decir, que a menor compromiso vasculoneural por parte del tumor, mayor posibilidad de erradicación tumoral.

También se ha analizado si los datos preoperatorios de la escala de Karnofsky influyen en el tipo de cirugía practicado y se ha visto que no hay significación estadística ( $\mathrm{p}<$ $0,9)$.

\section{Sistema de valoración pronóstica}

Basándonos en el grado de extirpación tumoral y en el del pronóstico postquirúrgico de los pacientes se han obtenido una serie de datos que nos han permitido la elaboración de una gradación que sirve de guía para conocer qué meningiomas de la base de cráneo pueden o no ser extirpados en su totalidad (Fig. 1). Dicha gradación ha sido la siguiente:

Grado I. Meningiomas de la base de cráneo que no afectan a pares craneales ni arterias o sólo afectan a un par craneal o engloban sólo una arteria. En estos casos la extirpación total ha sido del $98,3 \%(\mathrm{p}<0.0001)$ y las perspectivas de estos pacientes de alcanzar 70 puntos o más en la escala de Karnofsky fue de un 96,5\%. (Fig. 2).

Grado II. Meningiomas de la base de cráneo que afectan a un solo par craneal e incluyen una o más arterias. La extirpación total de estos tumores fue del $83,3 \%(p<0.0001)$ y alcanzaron 70 o más puntos en la escala de Karnofsky el $70,6 \%$ de los pacientes.( Fig. 3).

Grado III . Meningiomas de la base de cráneo que afectan a dos o mas pares craneales e incluyen varias arterias. La resección total de estos tumores desciende a un $42,9 \%$ de los casos $(\mathrm{p}<0.0001)$ y el pronóstico de estos pacientes de alcanzar un Karnofsky de 70 puntos o más fue solamente de un $60 \%$ (Fig. 4).

\section{Discusión}

El tratamiento quirúrgico de los meningiomas de la base de cráneo se encuentra comprometido por dos factores relevantes. $1^{\circ}$ por la facilidad que tienen estos tumores, en 

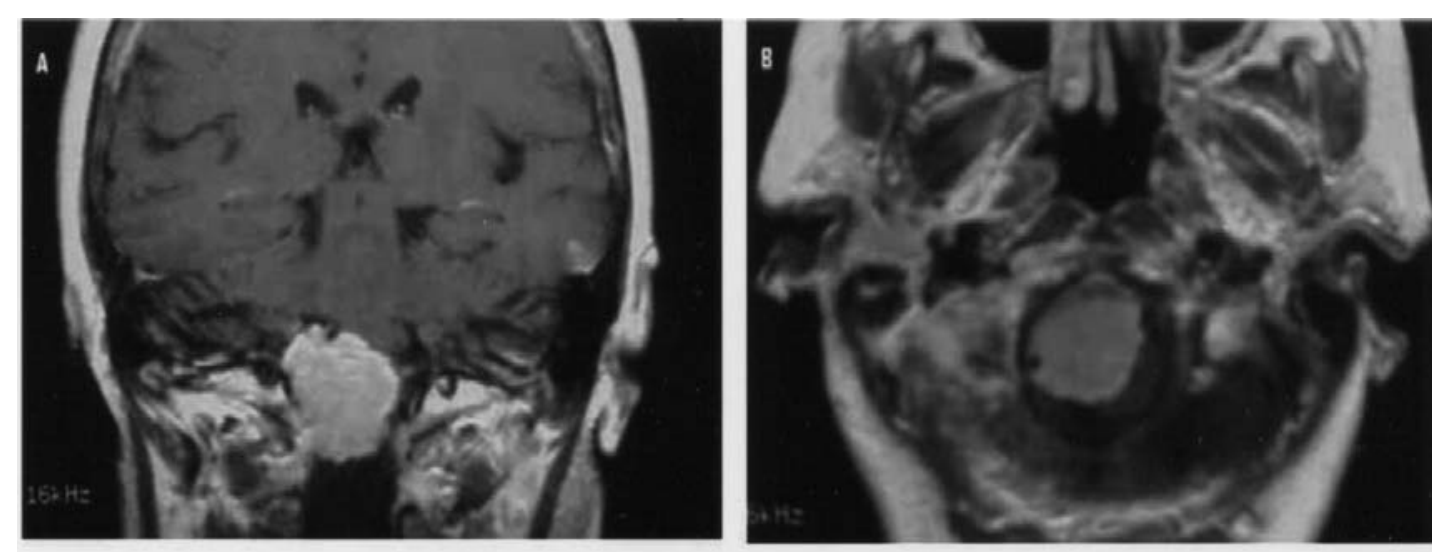

2005; 16: 477-485
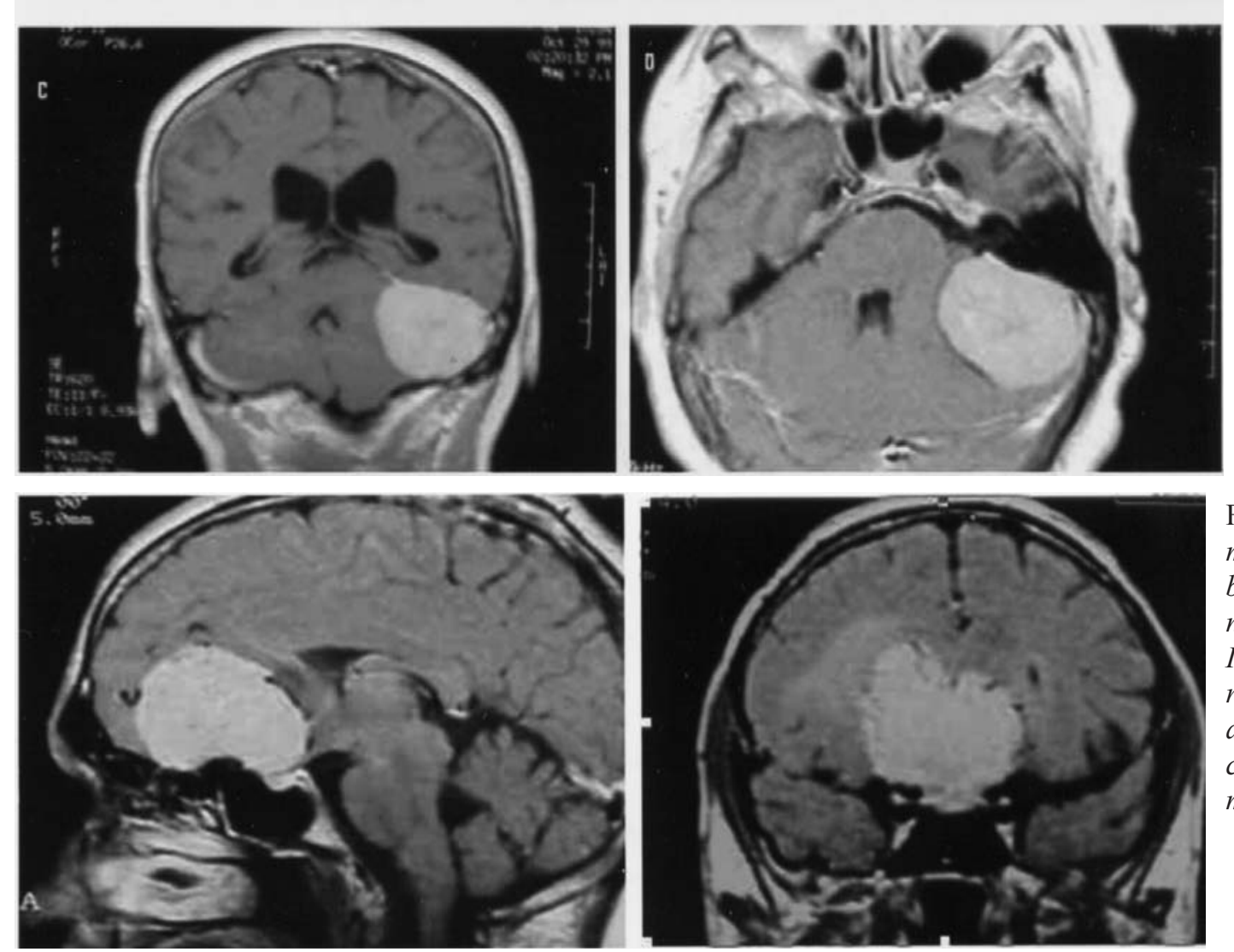

Figura 3. Grupo II : meningioma del tuberculum sellae. Clínica: afectación del II par craneal derecho e inclusión de ambas arterias pericallosas en la tumoración.
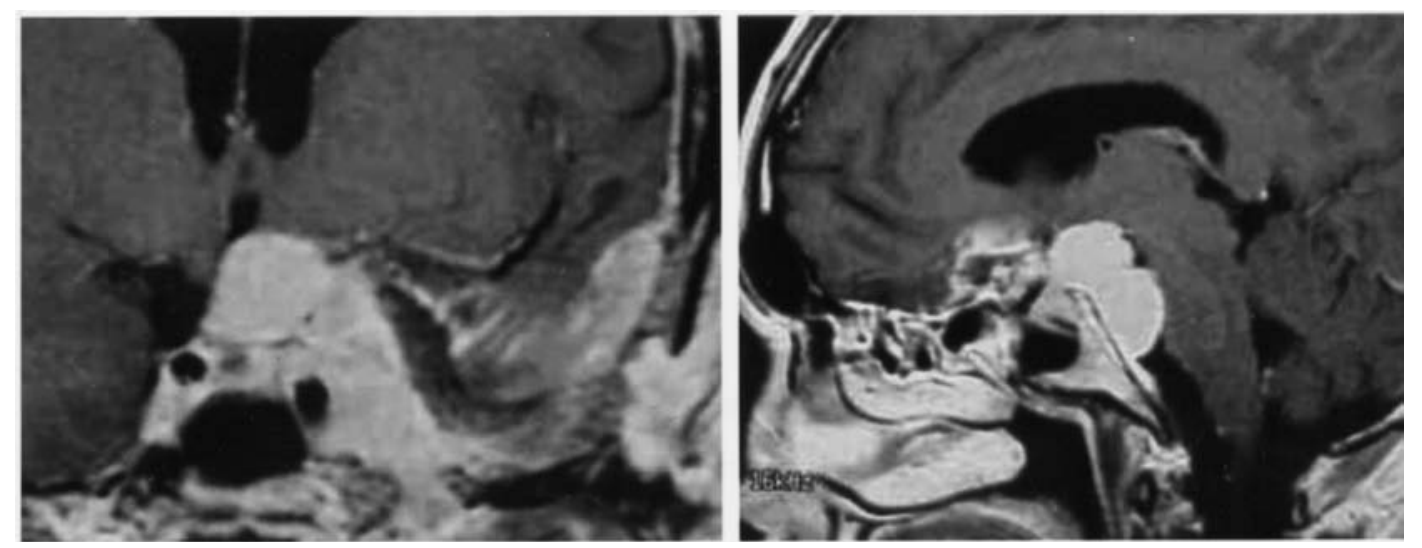

Figura 4. Grupo III: meningioma del ala interna del esfenoides izquierda con invasión de seno cavernoso. Clínica: afectación II, III, IV, VI y $1^{a}$ rama V pares izquierdos. Arteria carótida izquierda y su bifurcación englobadas por el tumor. 
su crecimiento, de englobar pares craneales y arterias de la base así como de comprimir el tronco del encéfalo y $2^{\circ}$ por la tendencia a las recidivas. Sólo hay que recordar que el Simpson, grado I, se asocia con un $9 \%$ de recidivas a los 10 años. Simpson grado II, un $19 \%$ a los 10 años y grado III, el 29\% a los 10 años $^{24}$. Ambos factores, crecimiento y recidiva, desde el punto de vista de una estrategia quirúrgica, son contradictorios. El primero, invita a ser cautos y nos sugiere limitar nuestras maniobras quirúrgicas para evitar secuelas; el segundo factor, las recidivas, nos inclinan a ser más agresivos. Entre estos dos extremos existen una amplia gamma de actitudes que han creado un importante estado de confusión a la hora de decidir un tratamiento Ante este dilema, la pregunta estaría: ¿Cuál debe ser nuestra estrategia quirúrgica ante un meningioma de la base de cráneo? ¿Se debe extirpar totalmente con el riego de secuelas o ser más conservador y hacer un tratamiento con radiocirugía? ¿De qué va depender nuestra decisión? De acuerdo con nuestros resultados, consideramos de interés el recordar que la posibilidad de ser más o menos radicales va a estar en relación con la proporción de arterias y pares craneales afectados por la tumoración. Creemos que la gradación, en tres grupos, que se presenta en este trabajo refleja con claridad lo que muchos neurocirujanos, de acuerdo con su larga experiencia, tienen en mente, es decir que la participación de pares craneales y arterias en el tumor, hace mucho más complicada su extirpación. Por lo tanto esa experiencia, mediante este método de aplicación analítica, se ha hecho algo más tangible y cercano a la realidad.

De acuerdo con los resultados de nuestra serie de 85 pacientes, los enfermos que presentan un meningioma de la base de cráneo que no compromete a pares craneales ni arterias o sólo engloba a un par craneal o sólo a una arteria, las posibilidades de que puedan extirparse totalmente se elevan a un 98,3\% y con unas perspectivas de alcanzar un Karnofsky de 70 o más puntos, en un 96,5\%. En este tipo de meningiomas la sugerencia es que se debe encaminar su tratamiento quirúrgico hacia una resección total. Por el contrario, cuando en la exploración neurológica y en los exámenes de imagen se detecta la presencia de pares craneales $\mathrm{y}$ arterias englobados por el meningioma, nuestra actitud, en estos casos, debe ser más prudente. Se debe realizar un detenido reconocimiento de los pares y arterias afectados, valorar nuestra experiencia quirúrgica e intentar la extirpación tumoral a sabiendas tanto por parte del paciente como por los familiares de que el porcentaje de extirpación total va a estar inversamente proporcional con el número de arterias y pares craneales afectados por el tumor. En estos casos hay que tener en mente que el tratamiento quirúrgico tendrá que completarse, en un alto porcentaje de casos, con radiocirugía $8,10,11,15,20$.

Valor del Sistema de Gradación. El resultado de nuestros estudios estadísticos ha permitido la posibilidad de presentarlos en un sistema compuesto por tres grados. Cada uno de ellos, partiendo de datos preoperatorios, nos orienta, con unas probabilidades de error de $\mathrm{p}<0.0001$, a conocer las perspectivas de una extirpación total o parcial de los meningiomas asentados en la base del cráneo.

En el Grupo I, las posibilidades de llevar a cabo una extirpación total del meningioma, se sitúan en un 98,3\%, descendiendo progresivamente a un $83,3 \%$ en el grupo II y a un $49,2 \%$ en el grupo III. Todo ello dependiendo, por razones de tratamiento estadístico, de la proporción de arterias y sobre todo de los pares craneales englobados por el tumor.

Esta clasificación, además de darnos una importante orientación quirúrgica, también, nos da una idea, bastante ajustada, sobre el pronóstico postoperatorio del paciente de acuerdo con el grupo en el que dicho paciente ha sido incluido. Cuando un enfermo pertenece al grupo I, sus posibilidades postoperatorias de alcanzar 70 puntos o más en la escala de Karnofsky es de 96,5\%, descendiendo, en el Grupo III, al 60\%. Basándonos en estos datos, se puede decir que, sobre las posibilidades de extirpación tumoral, existe una relación lineal inversa: a más arterias y nervios periféricos envueltos por el tumor, menos son las posibilidades de realizar una extirpación total de la tumoración. Sin embargo, en cuanto al porcentaje de extirpación total y pronóstico, la relación es directa, de acuerdo a cada grupo. Otro grupo de neurocirujanos ${ }^{18}$ ha realizado un estudio parecido al nuestro, diferenciándose en matices como la inclusión, entre sus variables, de meningiomas que habían sido anteriormente irradiados o la introducción de sólo tres pares craneales, III, V y VI, eludiendo los de fosa posterior, o la no introducción de una invasión ósea. Con un paquete estadístico similar, también han llegado a las mismas conclusiones: a menor número de variables incluidas en el examen preoperatorio del paciente, más posibilidades de extirpación total y mejor pronóstico de los enfermos.

Otra de las variables analizadas, la localización del tumor tuvo, también, un valor estadísticamente significativo $(\mathrm{p}=0.03)$ aunque éste se encontró muy por debajo al valor de las arterias y pares craneales incluidos en el tumor $(\mathrm{p}=0.001 \mathrm{y} \mathrm{p}=0.0001)$. Es bien conocido que aquellos meningiomas que asientan en la línea media de la base del cráneo, principalmente, procesos parasellares y de clivus ${ }^{4,6,21}$, presentan grandes dificultades a la hora de un probable planteamiento quirúrgico, pero también es importante recordar que la dificultad viene dada, no por la vía de acceso al tumor, es decir, no porque llegar hasta él sea la mayor dificultad, sino porque estos procesos en su crecimiento engloban arterias y pares craneales que son los que realmente plantean el verdadero problema a la hora de su total extirpación. En nuestra experiencia, cuando estos tumores aun no han englobado, de forma amplia, tanto a arterias como a pares craneales, ha sido posible su extir- 
pación total. Un ejemplo de ello son los meningiomas del agujero magno que dan clínica antes de alcanzar un gran tamaño y por esta razón, estos procesos, a pesar de una trabajosa vía de acceso, pueden ser extirpados sin mucha dificultad. En la realización de esta vía quirúrgica, lleva más tiempo las fases de abertura y cierre que la propia disección del meningioma.

Por todo lo dicho, creemos que el lugar del asentamiento de estos meningiomas tiene un claro valor, pero éste debe ser considerado, sólo desde un punto de vista localizador. Sin embargo, lo que nosotros hemos estado buscando y que consideramos tiene una mayor utilidad a la hora de montar una estrategia quirúrgica, es el valor predictivo. En este último, la presencia de arterias y pares craneales en la tumoración tiene una significación mucho mayor que el localizador, como lo demuestra el estudio estadístico.

El sistema predictivo comentado presenta tres importantes ventajas: una, la de ser su aplicación bastante simple. Consiste, solamente, en conocer el número de pares craneales afectados y la proporción de las arterias incluidas en la tumoración mediante un estudio de neuroimagen. La segunda ventaja consiste en poder predecir nuestras posibilidades de resección tumoral, basándonos solamente en datos de valoración preoperatorios y como tercera, es de interés resaltar que el estudio se basa en un valor estadístico lo que es sinónimo de evidencia científica.

Las aportaciones de otras escalas y clasificaciones a la neurocirugía han sido de indudable valor. Actualmente la $\operatorname{GCS}^{26}$ para los traumatizados craneoencefálicos, las escala de Hunt \& Hess $^{12}$ para los pacientes con hemorragias subaracnoideas, así como la de Spetzler \& Martin ${ }^{25}$ para el planteamiento quirúrgico de las malformaciones arteriovenosas cerebrales o como las incluidas en este trabajo de Karnofsky y Simpson ${ }^{14,24}$ están universalmente aceptadas y asumidas. Todas ellas han sido respaldadas por un estudio estadístico y son de una gran utilidad no sólo a médicos sino también a enfermos y familiares.

Notas de técnica quirúrgica. En el grupo I, la disección del tumor de los elementos que generalmente lo circundan, no suele presentar relevantes problemas. Sin embargo, cuando pasamos a los grupos II y sobre todo al grupo III, las circunstancias cambian y las posibilidades de una extirpación total, como ya se ha mencionado, van descendiendo progresivamente al encontrarnos con pares craneales y arterias englobados por la tumoración. Pero, la inclusión de estos últimos en la tumoración no entraña que haya que descartar totalmente una probable solución quirúrgica, ya que tanto en el grupo II como en el grupo III de nuestra serie, existe un 83,3 y $42,9 \%$ respectivamente de meningiomas que han podido ser extirpados en su totalidad. ¿Cual es la razón para que en estos dos últimos grupos exista la posibilidad de una extirpación total? Desde nuestro punto de vista, la experiencia del neurocirujano en reconocer, en el acto intraoperatorio, en qué plano meníngeo se está desarrollando la tumoración. Si la tumoración tiene un predominio de crecimiento aracnoideo, la disección del tumor, debido a la presencia de algunos planos de clivaje, tiene mas posibilidades de hacerse in-toto que si su crecimiento fuera totalmente aracnoideo o tuviera un predominio de desarrollo epidural ${ }^{27}$. En este último caso, en el epidural, no existen planos aracnoideos de clivaje, se acompaña de hiperostosis, hay afectación de los orificios de la base de cráneo e invasión de los pares craneales que salen por ellos, así como de la carótida, haciendo difícil la extirpación, sin dejar importantes secuelas neurológicas. En algunos de estos enfermos se pudo extirpar el tumor, pero fue complicado resecar la duramadre y casi todos tuvieron que ser incluidos en un grado II o III de Simpson, ya que incluso el coagular la dura entrañaba la posibilidad de lesionar algún par craneal. En aquellas otras ocasiones en que la tumoración tuvo un crecimiento, con gran proliferación tumoral de las tres capas, aracnoidea, dural y epidural, como ocurrió en varios de los meningiomas incluidos en el Grado III, el número de pares, arterias y venas que compromete se incrementa de forma significativa, dejando poco margen para llevar a cabo una cirugía radical sin que exista un importante déficit neurológico. De todas formas, hay que recordar que, fundamentalmente, las arterias son unas buenas estructuras de referencia y en varias ocasiones, un buen plano de clivaje para poder realizar la disección de estos tumores de la base de cráneo.

De todo ello se deduce, que los meningiomas de la base, incluidos en el Grupo I van a presentarnos planos de clivaje aracnoideo que nos permitirán su radical extirpación. Sin embargo, a medida que los meningiomas son incluidos en los grupos II y III de nuestra gradación, los planos de disección se hacen cada vez mas difíciles de encontrar, la probabilidades de infiltración tumoral aumentan y, por lo tanto, la posibilidad de una extirpación total disminuye, lo que nos obligará a completar su tratamiento con radiocirugía.

Referente al grado de extirpación quirúrgica de este tipo de meningiomas, en alguna serie ${ }^{18}$, se considera como resección total, sólo a los grados I y II de Simpson. Sin embargo, en este trabajo hemos seguido puntualmente los grados definidos por Simpson, en 1957, en su escala y consistentes en: Grado I, extirpación total, incluida duramadre y hueso afectados. Grado II, extirpación total del tumor con coagulación de la duramadre y Grado III, extirpación total del tumor sin resección o coagulación de la duramadre. Por lo tanto, los grados I, II y III han sido considerados como extirpación total del tumor. Otra consideración es evaluar el tipo de extirpación desde el punto de vista de la recidiva de estos tumores, pero ello no es el objetivo de este trabajo.

Finalmente, mencionar que el número de casos de nuestra serie, aunque suficiente como para tener un valor estadístico, aun puede ser considerado como exploratorio, 
por lo que futuros trabajos sobre el tema, con un mayor número de casos, podrán confirmar la utilidad de este sistema predictivo que, basándose en datos exclusivamente preoperatorios, nos orienta no sólo sobre las posibilidades de resección de estos tumores sino también sobre su pronóstico. Desde nuestra óptica, esta clasificación tiene el importante valor de ser una buena guía, tanto técnica como ética, para neurocirujanos, enfermos y familiares.

\section{Conclusiones}

El sistema de gradación que se propone, basado exclusivamente en datos preoperatorios de exploración neurológica y estudio de imágenes, permite, en los casos de meningiomas asentados en la base de cráneo, predecir tanto la posibilidad de una extirpación total o parcial como conocer el pronóstico quirúrgico de estos enfermos. Con la aplicación de este sistema podremos reducir la morbilidad, anticipar las necesidades de una terapia con Radiocirugía y dar una información al paciente y familiares sobre nuestro planteamiento quirúrgico.

\section{Bibliografía}

1. Adegbite, A.B., Khan, M.I., Pain, K.W., Tan, L.K.: The recurrence of intracranial meningiomas after surgical treatment. J. Neurosurg 1983; 58: 51-56.

2. Al-Mefty, O., Holoubi, A., Rifai, A., Fox, J.L.: Microsurgical removal of suprasellar meningiomas. Neurosurgery 1985; 16: 364-372.

3. Al-Mefty, O.: Clinoidal meningiomas. J. Neurosurg. 1990; 73: 840-849..-

4. Blanco, A., Morales, F., Hernández, J., Maillo, A., Díaz, P., Gomez Moreta, J., Gonsalves, J.M., Onzain, I.: Meningiomas de la región parasellar. Neurocirugía 1999; 10 : 119-130.

5. Bonald, JP., Thibaut, A., Brotchi, J., Born, J.: Invading meningiomas of the sphenoid ridge. J. Neurosurg. 1980; 53: 587-599.

6. Cushing, H., Eisenhardt, L.: Meningiomas: Their classification, regional behaviour, life history and surgical end results. Sprinfield, Charles C Thomas, 1938.

7. DeMonte, F., Smith, H.K., Al-Mefty, O.: Outcome of aggressive removal of cavernous sinus meningiomas. J. Neurosurg. 1994; 81: 245-251.

8. Duma, C.M., Lunsford, L.D., Kondziolka, D., Harsh, G.R., Flickinger, J.C.: Stereotactic radiosurgery of cavernous sinus meningiomas as an addition or alternative to microsurgery. Neurosurgery 1993; 32: 699-705.

9. Haddad, G., Al-Mefty, O.: Meningiomas: an overview. En ( Wilkins, R.H.: Rengachary, SS (ed). Neurosurgery, Vol. I, $2^{\mathrm{a}}$ ed. New York; McGraw-Hill, 1996; pp 833-841.

10. Hakim, R., Alexander, E. III., Loeffler, J.S., Shrieve,
DC., Wen, P., Fallon, M.P., Stiege, P.F., Black, P.M.: results of linear accelerator-based radiosurgery for intracranial meningiomas. Neurosurgery. 1998; 42: 446-454,

11. Hudgins, W.R., Barker, J.L., Schwartz, D.E., Nichols, T.D.: Gamma knife treatment of 100 consecutive meningiomas. Stereotact. Funct. Neurosurg. 1996; 66( suppl 1) : 121-128,

12. Hunt, W.E., Hess, R.M.: Surgical risk as related to time of intervention in the repair of intracranial aneurysms. J. Neurosurg. 1968; 28: 14-20.

13. Jaaskelainen, J.: Seemingly complete removal of histological benign intracranial meningiomas: Late recurrence rate and factors predicting recurrence in 657 patients - A multivariate analysis. Surg Neurol 1989; 26 : 461-469.

14. Karnofsky, D., Burchenal, J.: En Macleod CM (ed) : Evaluation of Chemotherapeutic Agents. New York, Columbia University Press, 1949, pp 191-205.

15. Kondziolka, D., Lundsford, L.D., Coffey, R.J., Flickinger, J.C.: Stereotactic radiosurgery of meningiomas. J. Neurosurg. 1991; 74: 552-559

16. Kotapka, M.J., Kalia, K.K., Martínez, J., Sekar, L.N.: Infiltration of the carotid artery by cavernous sinous meningioma. J. Neurosurg. 1994; 81: 252-255.

17. Leonetti, J.P., Reichman, H., Smith, P.G., Grubb, R.L., Kaiser, P.: Meningiomas of the lateral skull base: Neurotologic manifestation and patterns of recurrence. Otolaryngol Head Neck Surg. 1990;103: 972-980.

18. Levine, Z.T., Buchanan, R.I., Sekhar, L.N., Rosen, C.H.L., Wright, D.C.: Proposed Grading system to predict the extent of resection and outcome for cranial base meningiomas. Neurosurgery 1999; 45: 221-230.

19. Lobato, R.D., Alday, R., Gómez, P.A., Sánchez Aiceto, G., Ramos, A., González, P., Rivero, B., Rodríguez, C.: Meningiomas del ala esfenoidal. Estudio de 90 casos. Neurocirugía 1999; 10: 13-26

20. Lundsford, L.D.: Contemporary management of meningiomas: radiation therapy as an adjuval and radiosurgery as an alternative to surgical removal? J. Neurosurg. 1994; 80: 187-190.

21. Mirimanoff, R.O., Dosoretz, D.E., Linggood, R.M., Ojeman, R.G., Martuza, R.L.: Meningiomas: Analysis of recurrence and progression following neurosurgical resection. J. Neurosurg. 1985; 62: 18-24.

22. Pan, D.H.C., Guo, W.Y., Chung, W.Y., Shiau, C.Y., Liu, R.S., Lee, L.S.: Early effects of gamma knife surgery on malignant and benign intracranial tumours. Stereotact. Funct. Neurosurg. 1995; 64 ( suppl)19-3 1.

23. Pieper, D., Al-hefty, O.: Management of intracranial meningiomas secondarily involving the infratemporal fossa: Radiographic characteristics, pattern of tumor invasion, and surgical implications. Neurosurgery 1999; 45: 231-238.

24. Simpson, D.: The recurrent of intracranial meningiomas after surgical treatment. J. Neurol. Neurosurg. Psychiatry 
1959; 20: 22-39.

25. Spetzler, R.F., Martin, N.A.: A proposed grading system for arterio-venous malformations. J. Neurosurg. 1986; 65: 476-483.

26. Teasdale, G., Jennett, B.: Assessment of coma and impaired consciousness: A practical scale. Lancet 1974; 2: 8184 ,.

27. Yasargil, M.G.: Meningiomas of basal posterior cranial fossa. En Advances and Technical Standards in Neurosurgery. Spring-Verlag. Wien. New York Vol.,7, 1980 ; pp.107-108.

Comentario al trabajo Meningiomas de la base de cráneo. Un sistema predictivo para conocer las posibilidades de su extirpación y pronóstico de Morales y cols.

Los sistemas de predicción son muy útiles tanto para el neurocirujano como para el paciente y su familia. Una cualidad que se le pide a cualquier sistema de predicción es que sea universal, es decir, que se pueda utilizar no importa donde y por supuesto que sea sencillo. El sistema que proponen Morales y cols es muy simple y su bondad vendrá con la aplicación prospectiva en una serie ajena.

Algunos comentarios me vienen a la mente, como que no todos los pares craneales tienen el mismo rango, es decir, no creo que un nervio olfatorio determine tanto el resultado funcional como un nervio óptico. No sé cómo se podría remediar esto, tal vez se debería dar categorías a los nervios. Tampoco tiene el mismo valor una arteria cerebral media que una PICA. En la cirugía de la base de cráneo muchos factores se unen para producir el resultado clínico. Las asociaciones entre esas variables son, a veces complejas: pueden estar relacionadas entre sí, así como con el resultado clínico; el efecto de una puede ser modificado por la presencia de las otras; y los efectos conjuntos de
Morales, F.; Maillo, A.; Díaz-Álvarez, A.; Merino, M.; Muñoz-Herrera, A.; Hernández, J.; Santamarta, D.: Meningiomas de la base de cráneo. Un sistema predictivo para conocer las posibilidades de su extirpación y pronóstico. Neurocirugía 2005; 16: 477-485.

Correspondencia postal: F. Morales Ramos. Servicio de Neurocirugía. Hospital Universitario. Paseo de San Vicente, 58182. 37007 Salamanca.

dos o más pueden ser mayores que la suma de sus efectos individuales. El análisis multivariado es un método para considerar simultáneamente los efectos de muchas variables. En general, el análisis multivariado se emplea para ajustar (controlar) simultáneamente los efectos de muchas variables, para poder determinar los efectos independientes de una sola variable ${ }^{1}$.

No obstante, el sistema propuesto por Morales y cols nos puede servir de reflexión sobre nuestros resultados y también para informar a los pacientes y a sus familiares.

\section{Bibliografía}

1. Fletcher, R.H., Flettcher, S.W., Wagner, E.H.: Clinical Epidemiology: the essentials. Williams and Wilkins. $2^{\text {a }}$ edición. Baltimore. 1986. pp 105-126.

J. Vilalta Barcelona 